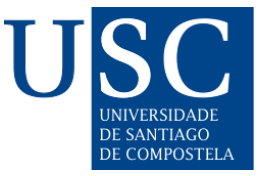

\title{
LA CORRELACIÓN MUTUA ENTRE EL CIBERESPACIO, LA POLÍTICA Y LO POLÍTICO: EL CASO DE LAS ELECCIONES PRESIDENCIALES MEXICANAS DE 2006,2012 Y 2018
} THE MUTUAL CORRELATION BETWEEN CYBER-SPACE, POLITICS AND THE POLITICAL: THE CASE OF THE MEXICAN PRESIDENTIAL ELECTIONS OF 2006,2012 AND 2018

\author{
Ernesto Ángeles Guerrero \\ Universidad Nacional Autónoma de México, México
}

Recibido: 21/10/2020; Aceptado: 12/01/2021

\section{Resumen}

Actualmente el ciberespacio y el internet están en prácticamente todo el mundo, aún en países de mediano y poco desarrollo, esta capacidad de penetración ha conducido y ha generado cambios sustanciales en las capacidades y ejercicio de poder por parte de actores estatales y no estatales, así como también dentro de la sociedad; sin embargo, el propio ciberespacio está condicionado por el ejercicio político de entidades políticas.

Esta tensión se traduce en herramientas nuevas usadas en prácticas viejas o en prácticas nuevas impulsadas por el ciberespacio y adaptadas a un contexto e intereses en específicos.

El caso de las elecciones federales en México a lo largo de tres periodos presidenciales da cuenta de la relación mencionada anteriormente, ya que muestra la simbiosis entre el ciberespacio y el sistema político y social, con todas sus limitantes técnicas, sus intereses detrás y una serie de pautas de ejercicio político propias de México, las cuales coinciden con la limitación de opciones que brinda el ciberespacio.

A su vez, el desenlace en la última elección federal (2018), y la conclusión de este escrito, muestra la no correlación con la linealidad de un posible argumento que simplifique la relación ciberespacio-política a manera de "mayor capacidad e inversión en el ciberespacio = mejores resultados o mayor capacidad de determinación en los resultados”.

Palabras clave: Ciberespacio; Política Mexicana; Elecciones Federales Mexicanas; Sistema Político; Política; Protección de Datos Personales; Democracia y Ciberespacio; Política y Ciberespacio; Elección Presidencial Mexicana 2018.

\begin{abstract}
Currently, cyberspace and the internet are practically all over the world, even in countries of medium and low development, this penetration capacity has led and has generated substantial changes in the capacities
\end{abstract}


and exercise of power by state and non-state actors, as well as also within society; However, cyberspace itself is conditioned by the political exercise of political entities.

This tension translates into new tools used in old practices or new practices driven by cyberspace and adapted to a specific context and interests.

The case of the federal elections in Mexico over three presidential periods gives an account of the aforementioned relationship, since it shows the symbiosis between cyberspace and the political and social system, with all its technical limitations, its interests behind and a series of political exercise guidelines of Mexico, which coincide with the limitation of options offered by cyberspace.

Also, the outcome in the last federal election (2018), and the conclusion of this paper, shows the noncorrelation with the linearity of a possible argument that simplifies the cyberspace-political relationship in the form of "greater capacity and investment in cyberspace = better results or greater ability to determine results".

Keywords: Cyberspace; Mexican Politics; Mexican Federal Elections; Political System; Politics; Personal Data Protection; Democracy and Cyberspace; Politics and Cyberspace; Mexican Presidential Election 2018.

\section{INTRODUCCIÓN}

La aparición y expansión del ciberespacio, entre y al interior de los Estados, ha sido un fenómeno complejo y singular en la historia de la humanidad, ya que nunca antes había existido tanta información generada, consumida, almacenada, reproducida, transmitida y comercializada en el mundo.

Además de la cantidad de información y la flexibilidad en la comunicación, el ciberespacio ha generado sinergias y dotado de capacidades a actores estatales y no estatales con las cuales, la vigilancia, el control y modificación conductual pueden ser implementadas de maneras nunca antes vistas, así como también la mejora en procesos, administración del Estado y el mejoramiento de las condiciones de vida de las personas.

Una nueva lógica productiva y administrativa centrada en los datos (su captura, almacenamiento, análisis, explotación, transformación e implementación) existe gracias al ciberespacio y amenaza con generar cambios de distinto orden en múltiples actores, latitudes y escalas; todo depende de las capacidades de adaptación y transformación de éstos, así como de con qué fines de deseen usar y transformar los datos que se recaben acerca de las personas y todas sus actividades.

De manera general, el ciberespacio puede entenderse a partir de su estructura y sus procesos:

En la primera, de acuerdo a autores como Lawrence Lessig (2006), Ronfeldt David (1991) y Deibert Ronald J (2013), las capacidades y ejercicio de poder va más allá de voluntades o siquiera la conciencia de los usuarios; sino que recaen en una arquitectura física y digital compleja que funge como la estructura del ciberespacio (entre estos componentes se encuentran la tecnología; los cables e infraestructura distribuida geográficamente y las convenciones y protocolos de cómo funciona el componente lógico -intangible- de la estructura del ciberespacio).

En el segundo elemento del ciberespacio, los usuarios y actores, constreñidos bajo estructuras y prohibiciones de distintos tipos (conversación/discusión, de acceso, difusión, búsqueda, comportamiento, normas morales y de valores, entre otros), establecen vínculos y procesos entre sí, lo que, de acuerdo al diseño del ciberespacio y, específicamente del internet, da una sensación de horizontalidad en los usuarios y en el discurso e idealización del ciberespacio. 
Tales procesos no sólo dependen de las capacidades y correlaciones de poder e imposición, sino también, y materia de esta investigación, dependen de las condiciones, prácticas y sistemas políticos (entre algunos otros), de una entidad política en específico; así como de las relaciones de cooperación y conflicto que se desarrollen en el ciberespacio (a manera de escenario o herramienta), ya que no es lo mismo las posibilidades de cambio por medio del ciberespacio en Estados Unidos que en Venezuela, México o China.

Dentro de la relación política-ciberespacio, es importante denotar el impacto mutuo que hay, ya que, mientras la política condiciona algunas estructuras, relaciones, comportamiento, procesos y diseño; el ciberespacio ha comenzado a condicionar, bajo parámetros funcionales y tecnológicos, la manera en la cual la política es ejercida o llevada a cabo.

Esto abre la posibilidad a estudiar la cultura y sistema político a partir del estudio numérico y pormenorizado de la sociedad y sus distintos patrones digitalizados, así como el aumento de capacidades de actores políticos y económicos tales como la vigilancia, la manipulación discursiva y reflectiva, el estudio y manipulación conductual, entre otros.

Para ahondar en el argumento anterior, y con la intención de generar una discusión exploratoria del tema, este documento intenta analizar la diferencia efectiva entre el discurso e idealización de las capacidades políticas en y por el ciberespacio, y el condicionamiento que impone la cultura y el ejercicio político, más allá de discursos idealistas o utópicos sobre la capacidad de incidencia y transformación del ciberespacio.

El punto de partida de esta investigación es el entrono estatal-institucional mexicano y los casos de contravención de ciertos valores liberales gracias a las nuevas capacidades que brinda el ciberespacio, tal como el derecho a la privacidad y a la seguridad.

\subsection{Contexto de la relación Estado y Ciberespacio: entre el espionaje y la seguridad}

Es importante señalar que el caso de la relación ciberespacio- Estado en México ha contravenido la idea idealista y utópica acerca de un ciberespacio libre, plural, abierto y seguro, ya que como se verá, el gobierno mexicano ha hecho uso de las capacidades institucionales de seguridad e inteligencia del Estado para beneficiar intereses particulares de ciertos actores más allá del bien común y el interés nacional, sobre todo en las administraciones gubernamental de 2006 a 2012 y de 2012 a 2018.

El uso del ciberespacio hecho por ambos gobiernos se hizo por encima de derechos humanos, garantías individuales, libertad de expresión y con el objetivo expreso de lograr fines políticos tales como el espionaje de enemigos hasta las amenazas para frenar las actividades de adversarios o ciudadanos en contra de intereses de actores gubernamentales.

Cabe aclarar que el uso de herramientas de inteligencia y seguridad del Estado para llevar a cabo espionaje político no es algo nuevo dentro del sistema político mexicano , ya que actores históricos como Frida Kahlo, Diego Rivera y el premio nobel de literatura, Gabriel García Márquez, fueron espiados con fines políticos por parte del gobierno de ese momento; así como también artistas como Mario Moreno "Cantinflas" y Juan Gabriel, El Economista (2019).

La diferencia entre estos casos y los gobiernos de 2006 a 2018 son muestra de una mejora en las capacidades y alcance tradicional de las herramientas de espionaje a manos del Estado, debido 
en gran parte al aumento en la penetración del ciberespacio y de internet entre la población y la cartera de recursos de telecomunicación desplegada en el Estado mexicano.

\subsubsection{El espionaje estatal y el uso del ciberespacio en la administración calderonista (2006-2012)}

El uso e instrumentalización de las instituciones de seguridad e inteligencia de parte del gobierno mexicano con el fin de espiar a enemigos políticos por medio del ciberespacio data, de acuerdo a las declaraciones del ex secretario de gobernación bajo el gobierno del ex presidente Peña Nieto, Miguel Ángel Osorio Chong, desde el gobierno del ex presidente Felipe Calderón.

La utilización del espionaje del gobierno calderonista se distinguió por dos casos:

El primero en torno a la cooperación gubernamental en materia de espionaje con el gobierno de Estados Unidos, encabezado por Barack Obama, bajo la operación denominada Switch Access, tal cooperación permitía a agentes estadounidenses la estadía en territorio nacional y la existencia de dos plataformas aéreas (K.A.-300 y PC-12), supuestamente operadas por empresas civiles contratadas por el Departamento de Defensa de EE.UU, so pretexto de la lucha contra el crimen organizado, aún cuando tal cooperación no contaba con la aprobación total de la Secretaría de la Defensa Nacional, RT Noticias (2019). Tal operación tenía no sólo como objetivo a ciudadanos mexicanos, sino también abarcaba a civiles y diplomáticos de Rusia, China, Irán y Corea del Norte.

El segundo caso, y materia de esta investigación,es la compra del software conocido como Finfisher ${ }^{1}$, un software de espionaje diseñado por la empresa Anglo-alemana Gamma Group International y vendido en México por medio de la empresa Obses en una compra que ascendió a los \$109.3 millones MXN.

La compra del software se hizo por medio de la Procuraduría General de la República y los contratos de licitación entre el gobierno y Obses de México dataron en un período de 2007 a 2012 y ascendieron a 37 contratos sin licitación de por medio. Se sabe que también se adquirió un software de georreferencia (Hunter Punta Tracking/Locsys) por \$93.2 millones MXN.

El documento de investigación realizado por la organización Digital Rights (2015), hay indicios de que estos métodos se usaron también en las entidades de Chiapas, Coahuila, Quintana Roo, Puebla, Tamaulipas y Veracruz.

Pese a que los tiempos de contratación tienen desde 2007, no fue sino hasta 2013 cuando se supo de la adquisición y existencia de este software de espionaje.

A su vez, bajo la administración de Peña Nieto se supo que en 2014 el gobierno calderonista también adquirió otro software de espionaje llamado Remote Control System, también denominado Da Vinci, el cual era un un programa malicioso que una vez instalado permitía extraer todo tipo de información y grabar la actividad en el teclado. El programa malicioso fue desarrollado por una empresa italiana de nombre Hacking Team, la cual ofreció sus servicios a países como Rusia, Uzbequistán, Sudán Chipre o Egipto.

Otro software malicioso que se compró junto a Da Vinci fue Galileo, vendido también por Hacking Team al gobierno calderonista.

Las instituciones que adquirieron el software malicioso fueron el Centro de Investigación y Seguridad Nacional (CISEN), la Policía Federal y Petróleos Mexicanos (Pemex); también se tuvo noticia de gobiernos locales como el del Estado de México, Jalisco o Querétaro. De éstos, los montos 
ascendían a casi un millón 400 mil euros facturados por el CISEN, la Procuraduría General de Justicia del estado de México y el gobierno de Jalisco con pagos superiores a los 700 mil euros.

De acuerdo a la periodista Yara Villaseñor, la cooperación entre el CISEN y Hacking Team se dio en un "ambiente abierto y de colaboración", acompañado de órdenes operativas "de infectar 20 objetivos por semana -más de mil al año- de las cuales sólo se lograron realizar 18”, según Villaseñor (2017).

\subsubsection{El espionaje estatal y el uso del ciberespacio en la administración de Peña Nieto}

El gobierno de Peña Nieto se vio envuelto en una serie de escándalos debido a la compra y uso de software y otras tácticas dirigidas para la vigilancia en el ciberespacio hacia actores incómodos y adversos al gobierno.

Pese a que, según el entonces secretario de gobernación, Osorio Chong, haya dicho que la compra de Galileo y Da Vinci se efectuó desde la administración anterior, no fue sino hasta el sexenio de Peña Nieto que se intentó que el gobierno esclareciera el uso de instituciones del Estado para llevar a cabo espionaje en contra de su población.

Entre 2016 y 2017 el gobierno peñanietista tuvo que afrontar una serie de quejas y peticiones de acceso a la información con relación al descubrimiento de un programa de espionaje digital, el programa de nombre Pegasus fue vendido por la empresa israelí NSO Group por medio de Balam Seguridad Privada SA de CV a la extinta Procuraduría General de la República (PGR).

Se sabe que la compra y uso del programa fue realizado por al menos tres agencias federales por un monto de casi 80 millones de dólares; sin embargo. el monto de las operaciones individualmente dependía del número total de objetivos a investigar. .

Pegasus se distinguió por la capacidad de infiltración en los teléfonos inteligentes y otros aparatos para monitorear cualquier detalle de la vida diaria de una persona por medio de su celular: llamadas, mensajes de texto, correos electrónicos, contactos y calendarios. Incluso podía utilizar el micrófono y la cámara de los teléfonos para realizar vigilancia; el teléfono de la persona vigilada se convertía en un micrófono oculto, tal como lo expusieron los reporteros Ahmed Azam y Perloth Nicole (2017).

La lista de objetivos de Pegasus abarcó a la periodista Carmen Aristegui y su hijo Emilio; también, Daniel Lizarraga, Salvador Camarena, Rafael Cabrera y Carlos Loret de Mola; los defensores de Derechos Humanos del Centro Miguel Agustín Pro, Mario Patrón Stehanie Brewer y Santiago Aguirre; los políticos panistas Ricardo Anaya y Fernando Rodríguez Doval, así como el entonces senador por este partido, Roberto Gil Zuarth, entre otros.

\subsubsection{El gobierno de Andrés Manuel López Obrador, ¿el fin de la vigilancia?}

Desde su candidatura de 2018 en el bloque Morena-PT-PES, el ahora presidente Andrés Manuel López Obrador, se quejaba acerca de la labor de investigación llevada a cabo por el CISEN contra su persona, catalogándola en algunos momentos de persecución política; por tanto, como propuesta 
política promovió la desaparición del CISEN y el fin de la vigilancia política por medio de las instituciones del Estado.

No sólo fue AMLO quien se quejó de las actividades del CISEN, sino que también el candidato de la coalición PAN-PRD-MC, Ricardo Anaya, acusó al CISEN de ejercer vigilancia contra él con fines políticos. Tras ambas declaraciones el secretario de Gobernación de esa administración, Alfonso Navarrete Prida, reconoció que el Cisen estaba pendiente de las actividades de los aspirantes a la presidencia, aunque negó que lo hiciera con el objetivo de espiarlos, sino que fue con el objetivo de proteger a actores de vital importancia en la vida política de México.

Después del triunfo de AMLO éste mencionó su interés en hacer efectiva la desaparición del CISEN y la creación de una nueva agencia de inteligencia (Agencia Nacional de Inteligencia) que no llevara a cabo espionaje telefónico a opositores.

Tal pretensión se vio justificada con el descubrimiento de instalaciones dedicadas al espionaje encontradas en la Ciudad de México y pertenecientes a Pegasus, el programa adquirido por el gobierno de Peña a la empresa NSO Group.

No se sabe hasta este momento si el cambio de gobierno y de nuevos cuadros y actores políticos signifique el fin del uso de instituciones de seguridad e inteligencia del Estado hacia la población y actores en específico con el fin de llevar a cabo operaciones de espionaje (más allá de la ley) aunque, al menos en el discurso, esta es la intención del nuevo gobierno.

\subsubsection{La opacidad estatal y el espionaje gubernamental}

En distintos momentos en la historia contemporánea de México los gobiernos en turno han utilizado a las instituciones estatales como herramientas al servicio de intereses políticos, tal es el caso de la adquisición de herramientas de espionaje y vigilancia; sin embargo, dichas compras han quedado protegidas por otras instituciones con el objetivo de salvaguardar la información de contratación y uso ante el derecho de la población de acceder a la información.

Ejemplo de esto son los millones de documentos clasificados en diferentes administraciones federales pasadas bajo el pretexto de seguridad nacional o información relacionada a la seguridad, la cual, tras el asesoramiento de instancias como el Instituto Nacional de Transparencia, Acceso a la Información y Protección de Datos Personales (INAI), se clasificó como secreta durante los próximos años.

Entre estos se encuentran los contratos (128/13 y 020/15) con los cuales el CISEN adquirió el spyware Galileo a Hacking Team, con reserva hasta el 2027. De acuerdo a R. Flores Linaloe, el INAI instruyó al órgano de inteligencia modificar la justificación de la reserva. El INAI asesoró para que no se basara en el artículo 14 de la antigua Ley Federal de Transparencia -como lo había hecho-, sino en el 13 de la misma ley, en aras de dejar claro que la revelación de la información de Galileo podría conllevar riesgos, R. Flores (2018).

En esta misma lógica se enmarca el aumento de documentos clasificados en las últimas tres administraciones federales, las cuales son: Vicente Fox Quesada (2000-2006) con 3 millones 839 mil carpetas reservadas, Felipe Calderón Hinojosa (2006-2012) con ocho millones y, por último, Enrique Peña Nieto (2012-2018) con 16 millones 109 mil 762. 


\subsection{Presentación de caso}

A manera de estudio de caso, en el presente escrito se analizarán brevemente tres momentos electorales clave en la historia de México y su relación con el advenimiento del ciberespacio como escenario y herramienta política.

La primera unidad de análisis son las elecciones presidenciales de 2006, momento en el cual el ciberespacio, y más específicamente la web 2.0, fue utilizada por primera vez en unas elecciones federales; sin embargo, dada la poca penetración y capacidades computacionales, los ejemplos y evidencias disponibles son pocos.

El segundo momento es en 2012, cuando el ciberespacio y sus herramientas se utilizaron de forma más intensiva, sistemática y masiva y, más importante, las redes sociales fueron un escenario que marcaron un antes y un después en la incidencia de la vida política en el ciberespacio y viceversa.

Por último, se analizarán las elecciones de 2018, en las cuales el ciberespacio se mostró como un escenario y una herramienta consolidada y usada por todos los partidos, además del aumento sustancial en el número y variedad de internautas; sin embargo, si sólo centramos nuestra atención en la capacidad de transformación del ciberespacio, el desenlace difícilmente fue acorde a las apuestas, dinero y estrategias invertidas en ese proceso, por tanto, es importante analizar la incidencia de la cultura y procesos políticos en el ciberespacio.

\section{LOS PROCESOS ELECTORALES DE 2006 A 2018}

\subsection{Fundamentación y objetivo}

Esta investigación parte del entendido que, tal como lo establece la investigadora Pippa Norris (2002), la red "puede favorecer la participación política pero no transformarla de manera radical”, bajo esta premisa, también se pondrán a discusión presupuestos -demasiadooptimistas, tales como los que menciona el investigador Manuel Torres (2013), de los denominados "ciberoptimistas", tales como:

- El ciberespacio impulsa la participación en asuntos políticos y suscita la circulación de información.

- Promueve las relaciones inter-grupales y, por tanto, se vuelve una herramienta fundamental en cuanto a la acción colectiva.

- Todo lo anterior asociado a la creencia que el ciberespacio genera aportes sustanciales al buen funcionamiento democrático y a una sociedad más libre, justa e informada.

El fin de este escrito es analizar la tensión mutua entre las dinámicas y herramientas que brinda el ciberespacio, su adopción y utilización por una determinada entidad política y los resultados que pueden devenir en consecuencias y convertirse en nuevos outputs para el ciberespacio nacional, el sistema y la vida política y su propia relación. 


\subsection{El ciberespacio en las elecciones federales de 2006}

El uso del ciberespacio en elecciones federales a nivel mundial tiene su origen en 2004 en Estados Unidos, cuando Al Gore y George Bush utilizaron el correo electrónico como plataforma de difusión de mensajes y propuestas en sus campañas políticas; sin embargo, sólo fue el preámbulo de lo que se avecinaría en los años sucesivos, ya que en ese momento el ciberespacio sólo sirvió como una herramienta de marketing muy básica que digitalizaba estrategias como el envío de publicidad por correo.

La utilización del ciberespacio en una elección federal en México fue a partir de las elecciones presidenciales de 2006, cuando la contienda se dio principalmente entre el candidato panista Felipe Calderón, el candidato de la Alianza por el Bien de Todos (APBT), Andrés Manuel López Obrador, y el candidato priísta, Roberto Madrazo.

En esta contienda electoral, de acuerdo a la Asociación de Internet, al 2007 existían alrededor de 23.7 millones de internautas, de los cuales el 79\% oscilaba en una edad de entre 18 y 34 años; de éstos, la mayoría (el 34\%) se conectaba en un promedio de 1 a 2 horas diarias. Y, de sus actividades sociales usuales en internet, se destacó el uso de correo electrónico (83\%), seguido del envío de mensajes instantáneos (74\%) y de visitar una sala de chat (64\%), Asociación de Internet, (2007). De ese número de usuarios, la mayoría (56.1\%) tenía una situación económica holgada.

Bajo este universo digital, las estrategias electorales se distinguieron por el uso del correo electrónico como medio de publicitar al candidato con el electorado; sin embargo, y a diferencia de las elecciones estadounidenses, el uso de ataques a la imagen de otros candidatos fue una norma dentro y fuera de internet, lo que refleja directamente la relación entre cultura política y la instrumentación de tecnologías y procesos que sirvan a diversos fines, más allá de ideales democráticos y utópicos.

En este sentido, el 8\% de la población total afirma que recibió correos por parte de partidos, la mayoría (45.2\%) aseveró haber recibido un mensaje favorable en favor del candidato del PAN, Felipe Calderón, frente a un 5.3\% de menciones en su contra; el candidato priísta, Roberto Madrazo, reportó un total de $24.2 \%$ de menciones positivas y un total de $13.7 \%$ de menciones negativas; mientras que AMLO, contrariamente a estos dos, presentó un 11.6\% de menciones positivas y un total de 32.2\% de mensajes con información negativa, así lo demuestra Meneses Rocha y Bañuelo Capistrán, (2009).

Lo anterior se desprende también del tipo de campañas que se decidieron hacer, ya que la campaña elaborada por el PAN apostó fuertemente por los medios masivos, y sobre todo la televisión, mientras que el candidato de la APBT optó por una campaña directa con los electores por medio de presentaciones en eventos públicos.

De acuerdo a la investigadora Meneses Rocha y a Bañuelo Capistrán (2009), (las elecciones federales de 2006) "en el plano político no crearon una agenda paralela ni aportaron información que los medios tradicionales a través de los spots no hayan dicho. Se limitaron a reproducir el eje del discurso negativo de los candidatos diseñado por sus estrategas".

Esto se puede entender debido a que en ese período, la exposición a medios como la televisión y el radio alcanzaban cifras del 98\% y del 70\%, respectivamente, frente al 31\% de exposición hacia el internet, según datos del Estudio sobre los hábitos de los usuarios de internet en México. Asociación Mexicana de Internet, (2007); lo que pone en evidencia la inmadurez y capacidad de alcance de la red en ese momento, y del ciberespacio en general. 
En ese proceso electoral el gasto total destinado a radio y tv ascendió a $\$ 2,580 \mathrm{mdp}$. Animal Político (2013, febrero 08), de este gasto la campaña de Calderón se destacó por ser la más costosa, ya que a los dos día de comenzada la contienda ya había hecho un gasto de 7 millones 343 mil 852 pesos. El Universal (2006, enero 23).

\subsection{El ciberespacio en las elecciones federales de $\mathbf{2 0 1 2}$}

Mientras que las elecciones presidenciales estadounidenses de 2004 marcaron el camino que seguirían los procesos electorales en otras latitudes, las elecciones federales estadounidenses de 2008 representaron un viraje en la forma de cómo se llevarían a cabo no sólo los procesos electorales, sino la política y sus capacidades cuantificables.

Este cambio se debió en gran parte a la mejora de las capacidades tecnológicas y a la constante evolución del ecosistema que es el ciberespacio, así como también al fortalecimiento e instalación de infraestructuras de comunicación.

Con esto se marcó el inicio de una compleja relación que apunta, no sólo a la profundización de la digitalización de servicios políticos, sino también al uso masivo de datos (la mayor parte de ellos personales) de múltiples formas y aplicaciones, la cuantificación emocional y conductual de los votantes y la generación de estrategias y contenido acorde a estas variables.

La campaña presidencial de Barack Obama en 2008 se caracterizó primeramente por ser llevada a cabo en un país con una penetración de internet del 75\%, de acuerdo a María Elena Meneses Rocha y Bañuelos Capistrán (2009), además, el uso del ciberespacio fue intensivo, participativo y flexible.

Dicha campaña no sólo se limitó a distribuir propuestas y la imagen del candidato, sino que llevó a cabo una estrategia que combinó el análisis de bases de datos, la conformación de comunidades en línea, la estructuración de una red de apoyos y donativos coordinados en la red, la comunicación bidireccional entre el equipo de Obama y los votantes, la estructuración de voluntarios y activistas autónomos, el uso combinado de sitios web y redes sociales, entre otras.

Las elecciones presidenciales de México en 2012 tuvieron lugar con estas lecciones dejadas por la campaña de Obama, bajo una experiencia como la de 2006, en un ciberespacio más maduro, con una específica cultura política como la mexicana y, sobre todo, tras dos sexenios panistas que no habían cumplido con las expectativas sociales, y que mostraban la incapacidad de resolución de problemas como la inseguridad y la violencia como resultado de la guerra contra el narcotráfico.

Para 2012 la penetración de internet había aumentado a 45.1 millones de usuarios nacionales con una población votante mayoritariamente compuesta por internautas de una edad que oscilaba de entre los 25 a 44 años.

En este año el tiempo de conexión promedio había aumentado a 4 horas diario, de entre las principales actividades que llevaban a cabo, la primera fue uso de correo electrónico (80\%); a diferencia de 2006, el uso de redes sociales apareció y se situó en la segunda actividad más realizada (77\%), seguida de la búsqueda de información (71\%) Asociación de Internet (2013).

En este contexto y en comparación con las elecciones de 2006, las redes sociales se posicionaron en un $92 \%$ de uso de los internautas, con un promedio de uso de 4 redes y con Facebook como la red social más utilizada ( $90 \%$ de los internautas inscritos); del total de usuarios de redes sociales, el 51\% accedió a publicidad política ,Asociación de Internet (2013).

En las elecciones de 2012 participaron el PAN, con su candidata Josefina Vázquez Mota. El PRI y el PRD lo hicieron en la Coalición Compromiso por México (PRI y PVEM) postuló a Enrique Peña 
Nieto, por último, la coalición Movimiento Progresista (PRD, PT y MC) nominó a López Obrador, el cual se presentó en su segunda elección a la presidencia.

Las elecciones de 2012 fueron para México lo que las elecciones presidenciales estadounidenses en 2008 fueron para el mundo.

A diferencia de las elecciones estadounidenses de 2008, las elecciones de México en 2012 sucedieron en un entorno político donde los bots y trolls ya existían como herramienta política en el ciberespacio, tal fue el caso de la elección gubernamental de Estado de México un año antes, en 2011. Islas (2018).

No sólo las elecciones de 2011 fueron un antecedente importante para 2012, sino que, y tras las lecciones que dejó 2006, el PRI, de entre los otros partidos, se distinguió por una preparación no sólo en la publicidad de medios (radio, tv y medios impresos), sino que desde 2007 implementó una estrategia web con un enfoque basado en las redes sociales (en ese momento la red utilizada fue Hi5).

En 2009 el diario británico The Guardian dio a conocer un documento en donde se hablaba de la colaboración de Televisa con el PRI en un proyecto para promover videos a favor de la campaña del PRI vía correos electrónicos masivos o en sitios como YouTube.

El nombre de la colaboración era "proyecto Handcock" y, en este, no sólo estaba inmiscuido el PRI y Televisa, sino que fueron asesorados por la empresa Blue State Digital (la cual trabajó con los demócratas en la icónica elección presidencial de Obama en 2008). Aristegui Noticias (2012, junio 26).

En ese momento la penetración de la televisión se mantenía muy por delante por sobre el internet, lo que añade importancia a la alianza PRI-Televisa.

Además, quien fue la coordinadora de la estrategia de la campaña de Peña Nieto en 2012, Alejandra Lagunes, fue asesorada por Blue State Digital, por lo que, al igual que la campaña de Obama, incorporó varias plataformas digitales como apoyo en la campaña, tales como Facebook, Twitter, YouTube, Google Plus (Google+), Instagram y Foursquare.

Cabe añadir que Lagunes antes es este puesto fungió como coordinadora de redes sociales de Eruviel Ávila, candidato vencedor a las elecciones gubernamentales de 2011.

La participación de jóvenes fue un elemento medular en la estrategia de campaña del PRI, ya que éstos fungieron como un elemento de posicionamiento y manipulación de la percepción en redes sociales.

La relación entre los jóvenes y su apoyo a Peña puede resumirse en dos casos: el movimiento "ectivista" y la creación de "PRIbook". El movimiento ectivista comenzó en diciembre de 2009 cuando un simpatizante priísta creó en Facebook una página de apoyo a Peña Nieto. Tres años después, este movimiento aglutinó a jóvenes que apoyaban a Peña y se organizaban para impulsar temas en Internet por medio de temas de tendencia en Twitter, según SonTusDatos, Artículo 12, A.C (2018, junio).

Por otro lado, en 2012 se creó "PRIbook", un sitio que concentró a las juventudes del PRI para apoyar en Internet a su candidato presidencial. En esa red social sólo los miembros del partido tenían acceso y se usaba para coordinar las acciones de apoyo a Peña Nieto en Facebook, impulsar un hashtag en Twitter o reaccionar a los ataques de los opositores. El registro en Pribook requería de una serie de datos personales básicos de los priístas, tal como nombre, dirección de correo electrónico y el sector del PRI al que estaban afiliados. ADN político (2012, octubre 06).

A su vez, Andrés Manuel, pese a pertenecer a la coalición Movimiento Progresista, creó una red social en torno a su Movimiento de Regeneración Nacional, MORENA (aún no reconocido como 
partido). No era necesario el crear una cuenta, aunque existía esa posibilidad, ya fuera directamente o por medio de alguna red social. En éste la interacción no estaba ligada a la autentificación por parte de una jerarquía de partido, y las características del sitio permitían la interacción entre usuarios.

En este proceso, los partidos con más presencia en las redes sociales eran el PRI (21, 666 usuarios de Facebook y 2, 662 usuarios de Twitter), el PAN (1,341 usuarios en Facebok y 23, 663 en Twittter), seguido del PRD (5, 213 usuarios de Facebook y 7, 900 usuarios de Twitter, de acuerdo a Animal Político (2011, septiembre 20).

Sin embargo, los candidatos con más seguidores estaban divididos, tanto por partidos, como por red social: Enrique Peña Nieto con 1, 008, 018 seguidores en Facebook y Andrés Manuel López Obrador con 97, 274 seguidores en Twitter.

Las redes sociales no sólo generaron fenómenos y sinergias dentro de los partidos, sino que también impactaron en la actividad de los votantes; tal fue el caso del movimiento \#YoSoy132. Éste se coordinó, articuló y difundió, en parte gracias al ciberespacio ya que el movimiento se originó como una protesta estudiantil que buscaba la democratización de los medios de comunicación, la creación de un tercer debate entre los candidatos presidenciales y el rechazo a lo que a su parecer fue la imposición mediática del candidato priísta. Martínez (2012).

La interacción de este movimiento con los simpatizantes del PRI generó una serie de sinergias que se vieron enmarcadas por lo que el periodista Héctor De Mauleón señala:

"La novedad de las elecciones de 2012 fue que los candidatos crearon ejércitos digitales encargados de abrir frentes electorales en la red. Tuiteros a sueldo, cuentas falsas, militantes en línea, robots controlados porprogramas informáticos (con el fin de) crear temas de moda y mejorar la imagen del uno mediante el deterioro de desprestigio de otro". De Mauleón (2012).

En esta tónica, en marzo de 2012 se calculaba un total de 4,103,200 cuentas de seguidores de Peña Nieto en Twitter, de las cuales 2,480,000, es decir, 60.44\%, estaban activas. Para algunos expertos, entre el 20 y 30 \% de los seguidores de Peña en Twitter eran bots. Islas y Pimienta (2016).

Un actor clave en la campaña negra digital del PRI fue el estratega político J.J. Rendón, el cual trabajó en la campaña por un período que fue de 2011 a 2013, J.J. La importancia de este estratega reacae en la guerra sucia que desató en las redes por medio de la contratación del hacker Andrés Sepúlveda.

Andrés Sepúlveda es un hacker colombiano que en 2012 se dedicó, de acuerdo a su testimonio, a "hacer acciones de guerra sucia y operaciones psicológicas, propaganda negra, rumores, en fin, toda la parte oscura de la política que nadie sabe que existe pero que todos ven", según lo establecido por Robertson Jordan, Et. Al. (2016).

El costo de sus servicios para la campaña de Peña Nieto fue de 600,000 USD durante tres años. Entre los costos que ofrecía, había un paquete por 12,000 USD al mes. Con este paquete, un cliente contrataba a un equipo que podía hackear teléfonos inteligentes, falsificar y clonar sitios web y enviar correos electrónicos y mensajes de texto masivos.

La campaña presidencial de Peña Nieto es una clara muestra de cómo el ciberespacio y su uso con fines políticos había cambiado drásticamente en apenas seis años; sin embargo, dicho cambio estuvo enmarcado en una cultura política que refleja no sólo valores democráticos y participativos dentro de los electores, sino también la tendencia al uso de la guerra sucia en las campañas políticas y el recurrir a descalificaciones personales, contextuales, funcionales o de cualquier tipo. 


\subsection{El ciberespacio en las elecciones federales de 2018}

El paralelismo entre las elecciones presidenciales estadounidenses de 2016 y las elecciones mexicanas desde 2006, 2012 y 2018 es más evidente, no sólo por el factor cibernético y de datos inmiscuido, sino por la implementación de herramientas de campaña negra en Estados Unidos que no habían tenido el tamaño y alcance como las elecciones de 2016, y que contraviene el discurso democrático, participativo y plural de las redes.

Las elecciones presidenciales mexicanas de 2018 marcaron un hito dentro de la historia política nacional, ya que no sólo se caracterizaron ser el proceso electoral más grande de la historia moderna de México, sino también por ser llevadas a cabo en un contexto de hartazgo político y rechazo a lo que el votante consideraba como el sistema.

Además, el mercado digital y el ciberespacio presentaban opciones novedosas tal como la etno y la psico segmentación, ofrecidas por empresas, nacionales e internacionales, dedicadas a la recopilación y análisis masivo de datos, más allá de una estrategia de mailing (2006) o el uso masivo de redes sociales (2012), SonTusDatos. Artículo 12, A.C (2018).

El rasgo característico de estas elecciones, en comparación a las de 2012, es que la implementación de noticias falsas en las campañas electorales fue más ampliamente usada que los bots, sobre todo los digitales. Y, de acuerdo al reportero español, Fernando Peinado, "Los partidos han descubierto tácticas más efectivas como la generación de noticias falsas y publirreportajes en portales supuestamente informativos que se venden al mejor postor". No extraña esta deriva, ya que las noticias falsas se expanden "significativamente más lejos, más rápido, más profunda y ampliamente" que las verdaderas, según por lo escrito por Peinado (2018).

Para 2018 la penetración de internet dentro de la sociedad ascendió a 82.7 millones de usuarios, casi el doble de internautas que en 2012 y cuatro veces más que en 2006. De este número de internautas, la mayoría (el 40\%) está conformado por individuos de una edad entre 18 y 34 años. El nivel socioeconómico mayoritariamente expresado es el medio bajo (36\%), seguido del medio alto (32\%), Asociación de Internet (2018).

El tiempo promedio de conexión ascendió a 8 horas y 12 minutos, aunque, de acuerdo al 64\% de los internautas, se percibió que la conexión a internet constaba de 24 horas del día. El dispositivo favorito de conexión fueron los smartphones con un 89\% de preferencia y el acceso a redes sociales se posicionó en el primer lugar de las actividades online de los usuarios (89\%), seguido de Enviar y recibir mails (84\%) y participar en chats (83\%), Asociación de Internet (2018).

En este momento el acceso a redes sociales estaba dominado por Facebook (98\%), Whatsapp (91\%) y Youtube (82\%).

El proceso electoral presidencial de 2018 se dio entre el candidato de la coalición Juntos Haremos Historia (Morena, PT y PES), Andrés Manuel López Obrador; Ricardo Anaya, candidato de la coalición Por México al Frente (PAN, MC y PRD); el candidato de la coalición Todos por México (PRI, PVEM y Nueva Alianza) José Antonio Meade, y los candidatos independientes, Jaime Rodríguez Calderón, mejor conocido como "El Bronco" y Margarita Zavala, esposa del expresidente Felipe Calderón, esta última se retiró de la contienda antes de las elecciones.

Un elemento de continuidad lo representó el candidato de MORENA, Andrés Manuel López Obrador, el cual se presentó por tercera vez consecutiva en las elecciones, lo que le permitió tener una sólida base de apoyo y campaña acumulada, aunque también esto representó la reactivación de estrategias y un discurso ya existente, dentro y fuera del ciberespacio y que, de acuerdo a la visión 
de la oposición, había sido fundamental para cooptar el ascenso a la presidencia de AMLO los dos sexenios anteriores.

La presencia de los medios de comunicación tradicionales se mostró costosa y, si sólo nos guiamos por el resultado electoral, podríamos decir que poco efectiva, ya que, de acuerdo a las cifras del Instituto Nacional Electoral (INE), la coalición Juntos Haremos Historia, que resultó vencedora, recibió casi la mitad de tiempo en spots publicitarios $(4,591,836)$ frente a los 9, 003, 234 de la coalición Todos por México y los 8, 673, 468 de la coalición del candidato panista, Ricardo Anaya, de acuerdo a datos de Ávila Yuriria, (2018, abril 18).

En lo que respecta al ciberespacio, la contienda presidencial de 2018 se distinguió por una serie de irregularidades en los afiliados a los partidos, un fenómeno recurrente en el mal manejo de bases de datos de las instituciones; la presencia constante de noticias falsas en redes sociales y apps de mensajería instantánea, alimentadas y diseminadas por bots (orgánicos y digitales); la participación de empresas de servicios políticos novedosos; $y$, al igual que en las elecciones de 2006 y 2012, una constante opacidad en la determinación de gastos publicitarios en internet, sobre todo debido a la flexibilidad y versatilidad de creación y diseminación de contenido en la red y a la dificultad de auditar.

Durante el proceso de campaña los candidatos habían realizado inversiones considerables en publicidad en medios digitales, al sumar 141.9 millones de pesos. Meade se situó en el primer lugar con un gasto de 65.6 millones de pesos); seguido de Anaya, 63.8 millones; 'El Bronco', 5.5 millones de pesos y Margarita Zavala, con 7 millones en redes sociales, antes de abandonar la contienda, Rodríguez, (2018, junio 25). El único contendiente que afirmó no haber realizado ningún gasto en redes sociales fue AMLO.

Este dato resulta complejo si se tiene en cuenta que, de acuerdo a los datos del INE, hasta el 25 de mayo, los candidatos presidenciales habrían pagado por difundir 575 "anuncios" en páginas de internet y redes sociales: 292 de Ricardo Anaya, 171 de José Antonio Meade, 73 de López Obrador, 22 de Margarita Zavala y 17 de Jaime Rodríguez Calderón, Nicolai Aldo, Et.Al. (2018, junio 04).

La explicación ante tales irregularidades corre en dos vías, una general y la otra de estrategia de campaña de la coalición Juntos Haremos Historia.

La primera es, como ya se había mencionado, debido a la capacidad de fiscalización del Instituto Nacional Electoral (INE), ya que, de acuerdo al informe "Dinero bajo la mesa", publicado por Mexicanos Contra la Corrupción y la Impunidad (MCCI) y la consultora Integralia:

En palabras de Nicolai Aldo, Et.Al. (2018, junio 04), "El problema radica en que con frecuencia (el INE) carece de la infraestructura y de la inteligencia financiera para detectar el flujo total de recursos en efectivo que fluyen a las campañas y los gastos que se realizan", sin contar con que, de acuerdo al estudio, de cada peso declarado, en realidad el gasto asciende a 15, o más. Además que la contratación de servicios de internet se da por terceros y no por contratación directa, tal como lo dejó en claro Google ante el proceso electoral ${ }^{2}$ por esta razón, tampoco se encontró a Twitter ni Facebook en el Registro Nacional de Proveedores.

La segunda explicación viene de la estrategia que adoptó la coalición liderada por AMLO y coordinada digitalmente por la actual senadora de Morena, Tatiana Clouthier. Dicha estrategia se valió de la flexibilidad, espontaneidad y difusión de las redes para crear contenidos simples (memes, comentarios) y complejos (manuales, infografías), todo esto bajo la coordinación de la cuenta "Abre más los ojos", Esteinou, Javier, (2019, abril 05). 
En dicha campaña no sólo fueron los jóvenes los que llevaron las riendas del proyecto, sino que también miembros y partidarios importantes de Morena contribuyeron a la discusión y difusión digital, como en el caso de la propia Tatiana, John Ackerman y Epigemio Ibarra.

Pese a esto, una investigación realizada por el periódico Reforma reveló que Andrés Manuel López Obrador registró en Twitter la mayor cantidad de 'bots' (cuentas fantasmas o fraudulentas) con un millón 467 mil 500 cuentas falsas, Recillas, Angélica., (2019, enero 18); lo que equivale a un 67.6\% de bots, seguido de Meade Kuribreña con 59.8\%; Anaya Cortés, 49\%, y Rodríguez Calderón, 45.8\%, Aristegui Noticias, (2018, junio 20).

Aunado a este panorama, en las elecciones de 2018 el PRI y el PAN optaron, de nueva cuenta, por una estrategia basada en un discurso de descalificación y ataques personales, principalmente dirigidos a AMLO, lo que, a todas luces, ayudó a que la cuenta "Abre más los ojos" recibiera un total de 98 millones de impactos sólo en Facebook, Navarro, María Fernanda, (2019, julio 20).

Un resultado claro de esta apuesta es lo que menciona la investigadora Beatriz Fernández, (2018): "La clase política mexicana le apostó al miedo a AMLO como mensaje único, pero en un entorno de tal desprestigio del sistema, el mayor miedo del electorado era a la continuidad".

Dentro de la lógica de campaña sucia, un elemento que pudo añadirse a las elecciones pero que, debido a las características del electorado mexicano y al mercado de datos nacional, no sucedió, fue la colusión de la empresa de análisis de datos, Cambridge Analytica con el PRI.

Aunque las negociaciones comenzaron desde 2017 entre estos dos actores, la información no saldría a la luz hasta abril de 2018, tras conocerse el incidente de Cambridge Analytica y el uso indiscriminado y no consensuado de bases de datos en Facebook de ciudadanos, con el fin de generar contenido a la medida e impactar los resultados electorales, tal como lo presumían en el Brexit y en las elecciones presidenciales estadounidenses de 2016, Asad Ahmed y Hakim Danny, (2018).

La noticia resultó estremecedora para algunos investigadores, académicos y público en general, no sólo por la violación a la privacidad, sino por las capacidades que estaban desarrollándose y que podían cambiar radicalmente la forma en la que unas elecciones tuvieran lugar; sin embargo, y tras un breve análisis de la oferta nacional, se puede concluir que esta clase de servicios ya está desarrollándose y operando dentro del mercado nacional, aunque constreñidos bajo el marco legal nacional en materia de protección de datos personales. ${ }^{3}$

En México existen pocas empresas dedicadas al análisis de datos para partidos políticos, a diferencia de los servicios de comunicación y publicidad política. Las principales empresas mexicanas del sector ofrecen servicios de análisis histórico de datos electorales (tendencias de voto) y de datos estadísticos poblacionales (datos socioeconómicos, educativos y de carácter político), ambos con el fin de hacer un perfilamiento cruzado entre características sociales y decisiones electorales, SonTusDatos, Artículo 12, A.C. (2018).

Estas empresas también crean noticias y usuarios falsos para apoyar a un candidato; miden y rastrean la movilidad y la interacción de personas en eventos políticos para conocer el comportamiento social y realizar eventos más efectivos; y analizan variables socioeconómicas con el fin de tener una idea a nivel regional de qué características comparten los votantes y cómo captar sus necesidades. Dentro de estas empresas figuran Descifra (fusionada con Telus, otra empresa de análisis de datos), Klustera, Victory Lab (la cual, de acuerdo a su presidente, Carlos Merlo, se especializa en bots y noticias falsas y la cual tuvo su aparición en la escena electoral desde 2012), Chávez Gabriela, (2017). 
Pese a que en este proceso electoral no se suscitó un movimiento como YoSoy132, la iniciativa VerificadoMx fungió como un contrapeso ciudadano al uso indiscriminado de las redes sociales y de internet por parte de partidos políticos y servicios adyacentes. Dicha iniciativa surgió con el fin de desmentir información y noticias falsas y coadyuvó la participación de la iniciativa privada, medios de comunicación internacionales y a la ciudadanía organizada.

Pese a las estrategias, dinero, actividades y material invertido, los resultados electorales de 2018, no sólo presidenciales, le dieron una apabullante victoria a Andrés Manuel y a su partido, si el análisis partiera únicamente de la inversión e impacto tecnológico, no se podrían interpretar los resultados correctamente, ya que en este proceso la cultura política, el descontento social, la corrupción y diversos indicadores económicos fungieron un papel primordial que impactaría en los resultados finales más allá del peso de la tecnología.

\section{CONCLUSIÓN}

El impacto del ciberespacio dentro de diversas áreas del conocimiento usualmente viene acompañado de una suerte de lo que Evgeny Morozov (2011), en su libro The Net Delusion. apunta como determinismo tecnológico (la tecnología como solución a cualquier problema, aunque éste no sea de naturaleza tecnológica) y un discurso optimista que a veces raya en lo utópico, ya que se suele comparar el ciberespacio, específicamente el internet, con una herramienta que por sí misma impulsará a la democracia, la participación informada y las libertades sociales alrededor del mundo.

Sin embargo, debe tenerse en cuenta el amplio panorama de opciones y posibilidades que existen, ya que por un lado la tecnología afecta a la sociedad y, por el otro, la sociedad se apropia y adopta la tecnología a partir de sistemas de prácticas y ejercicios de poder establecidos a través del tiempo.

En muchos casos estas opciones pueden resultar en amenazas a la vida, ejercicio y sistema democrático, así como la posibilidad de aumentar el control social, disminuir la capacidad de transparencia o facilitar la manipulación a gran escala social o permitir y promover discursos segmentados potenciados por las cámaras de eco que resultan ser las redes sociales, con todo y discursos radicales y extremistas.

La relación mutua entre política el ciberespacio y la cuantificación de indicadores se puede ver más clara a la hora de analizar las elecciones, ya que en ellas es posible rastrear el efecto de la política en el ciberespacio (generación de grupos y comunidades políticas; los controles y accesos permitidos en el ciberespacio implementados por el gobierno, las discusiones políticas y sus vicios característicos -como el uso de noticias falsas o bots-, entre otros).

Y del ciberespacio en la política (la creación de bases de datos de votantes, pormenorizadas y segmentadas a partir de rasgos no sólo socioeconómicos, sino psicológicos y conductuales; el espionaje a otros candidatos; los peligros asociados a la manipulación política por medio de publicidad, mensajes y material personalizado -alimentado no sólo de las demandas sociales, sino de los miedos, aspiraciones y otros elementos psicológicos-, son algunos de los ejemplos).

México es muestra de cómo la utilización del ciberespacio puede tener un impacto negativo en los actores y procesos políticos y, a su vez, también es muestra de la adaptación tecnológica en una cultura política particular.

El análisis de las elecciones mexicanas puede dar cuenta de la importancia de la cultura política sobre el ciberespacio y la configuración de opciones disponibles, así como también de 
indicadores culturales y socioeconómicos. Además, en éste se puede notar la progresión y madurez del ciberespacio a través del tiempo y los riesgos asociados que pueden significar un retroceso sustancial en la cultura y sistema político de los Estados.

Aunque no sólo los gobiernos, partidos políticos y otros actores poderosos han hecho uso de los beneficios del ciberespacio para sus propios fines, sino que la ciudadanía, así como ciertos actores e iniciativas privadas, dan muestra del potencial de resistencia que puede tener el ciberespacio para la ciudadanía y la democracia en general.

Ejercicios ciudadanos como el movimiento YoSoy132 y VerificadoMx deberían de fomentarse día a día, ya que, tras el final de las elecciones, las estrategias políticas usualmente llegan para quedarse, como el caso de los bots, las noticias falsas y el uso masivo de datos personales.

Así como también la adhesión y ejecución a mecanismos internacionales de transparencia, anticorrupción, fiscalización, de derechos humanos y gobernanza de internet, entre otros.

\section{BIBLIOGRAFÍA}

“Antes de Pegasus existió Galileo: todo será confidencial hasta el 2027". Yara Villaseñor (Miércoles 28 de junio de 2017 ) http://www.laizquierdadiario.mx/Antes-de-Pegasus-existio-Galileo-todo-sera-confid encial-hasta-el-2027

“¿Cambridge Analytica en México? AMLO y Anaya piden investigar presunta intervención en electores” Animal Político (21 de marzo, 2018) https://www.animalpolitico.com/2018/03/cambridge-analytica -mexico-amlo-anaya/

"Caso Pegasus: Hacienda perdonó a la empresa que vendió el equipo de espionaje; también vigilaron a la viuda de Javier Valdez". Infobae $(20$ de marzo de 2019) https://www.infobae.com/america/mexico/2019/03/20/caso-pegasus-hacienda-perdono-a-l a-empresa-que-vendio-el-equipo-de-espionaje-tambien-vigilaron-a-la-viuda-de-javier-valdez/

"Cisen, Pegasus y un peligroso fetichismo". Salvador Camarena (Marzo 2019) https://www.elfinanciero.co m.mx/opinion/salvador-camarena/cisen-pegasus-y-un-peligroso-fetichismo

"Con el fin del Cisen, se acabará el espionaje telefónico: López Obrador". Excelsior (Septiembre 2018) 14/07/2018 https://www.excelsior.com.mx/nacional/con-el-fin-del-cisen-se-acabara-el-espionaje-t elefonico-lopez-obrador/1252335

“¿Cuánto costaron las elecciones de 2012?”. Animal Político. (2013, febrero 08). https://www.animalpoliti co.com/2013/02/cuanto-costaron-las-elecciones-de-2012/

“Desde Cantinflas y Frida Kahlo hasta John F. Kennedy, así espiaba el gobierno de México". El Economista (12 de abril de 2019) https://www.eleconomista.com.mx/politica/Desde-Cantinflas-y-Frida-Kahlo-hasta -John-F.-Kennedy-asi-espiaba-el-gobierno-de-Mexico-20190412-0024.html

"El Cisen: Inteligencia de Estado... que no fue". Erubiel Tirado (30 julio, 2018) https://www.proceso.com.m $\mathrm{x} / 544943 /$ el-cisen-inteligencia-de-estado-que-no-fue

"En México no hay regulación del espionaje: especialistas". El Sol de México (Mayo 2019) https://www.elsoldemexico.com.mx/mexico/justicia/en-mexico-no-hay-regulacion-del-espio naje-especialistas-3644842.html

“Felipe Calderón, el candidato que más spots transmitió el fin de semana”, en El Universal, (2006, enero 23) http://archivo.eluniversal.com.mx/nacion/134371.html

"FinFisher en México: Sonríe, te siguen espiando" Digital Rights LAC (Junio 2015) https://www.digitalrigh tslac.net/es/finfisher-en-mexico-sonrie-te-siguen-espiando/ 
"Hallan en México edificio oficial con equipo de espionaje". HispanTv (10 de diciembre de 2018) https://w ww.hispantv.com/noticias/mexico/395683/espionaje-edificio-estatal-pena-nieto

“Informe: México pagó \$15 millones a una empresa de ciberespionaje". HispanTv (4 de septiembre de 2016) https://www.hispantv.com/noticias/mexico/286935/mexico-paga-espionaje-millones-nso-group

"La sombra del Cisen se mete a la contienda de las \#Elecciones2018". Expansión (Febrero 2018) https://politica.expansion.mx/politica/2018/02/13/la-sombra-del-cisen-se-mete-a-la-contie nda-de-las-elecciones 2018

"Los 5 casos de espionaje del gobierno mexicano". Ruy Alonso Rebolledo (20 de junio de 2017) https://www.eleconomista.com.mx/politica/Los-5-casos-de-espionaje-del-gobierno-mexicano -20170620-0101.html

“Los bots y su influencia en la elección presidencial mexicana: Albert-László Barabási” Aristegui Noticias, (2018, junio 20). https://aristeguinoticias.com/2006/mexico/los-bots-y-su-influencia-en-la-eleccion -presidencial-mexicana-albert-laszlo-barabasi/

“Los expedientes secretos de Enrique Peña Nieto". Linaloe R. Flores (27 Noviembre 2018) https://www.vic e.com/es_latam/article/439a8n/los-expedientes-secretos-de-enrique-pena-nieto

"México, el principal cliente de una empresa que vende software para espiar". Arturo Angel (7 de julio 2015)https://www.animalpolitico.com/2015/07/empresa-de-hackers-exhibida-por-venta-de-s oftware-espia-a-paises-represores-y-mexico-resulta-su-principal-cliente/

“México espió para la CIA a diplomáticos y ciudadanos de Rusia, China y otros países". RT (20 ago 2019) htt ps://actualidad.rt.com/actualidad/324539-mexico-espionaje-cia-china-rusia

"PRI y PAN, los partidos con más presencia en redes sociales: AMIPCI". Animal Político, (2011, septiembre 20). https://www.animalpolitico.com/2011/09/pri-y-pan-los-partidos-con-mayor-presencia-en-la-r edes-sociales-amipci/

“¿Quieres amigos políticos? Da 'like' a Pribook o FaceMorena”. ADN político. (2012, octubre 06). http://stat ic.adnpolitico.com/2012/2012/10/03/quieres-amigos-politicos-dale-like-a-pribook-o-facemorena

“'Somos los nuevos enemigos del Estado': el espionaje a activistas y periodistas en México". Azam Ahmed y Nicole Perloth (19 de junio de 2017) https://www.nytimes.com/es/2017/06/19/mexico-pegasus-ns o-group-espionaje/

"Sospechan mayor espionaje del gobierno mexicano". Julio Sánchez Onofre (13 de febrero de 2017) https://www.eleconomista.com.mx/politica/Sospechan-mayor-espionaje-del-gobierno-mexic ano-20170213-0039.html

“The Guardian revela unidad secreta de Televisa para la campaña de EPN". Aristegui Noticias, (2012, junio 26). https://aristeguinoticias.com/2606/lomasdestacado/theguardian-revela-unidad-secreta-de-tele visa-para-la-campana-de-epn/.

Asad Ahmed y Hakim Danny "Los recursos del PRI en las presidenciales: de Cambridge Analytica a la politización de la justicia”. New York Times, (2018, junio 24). https://www.nytimes.com/es/2018/06 /24/pri-mexico-elecciones-pgr-trife/

Asociación de Internet. "Estudio sobre los hábitos de los usuarios de internet en México 2007” (2007). Consultado el 04 de junio 2019. https://www.asociaciondeinternet.mx/es/component/remository/Habitos-de-Internet/Estud io-sobre-los-habitos-de-los-usuarios-de-internet-en-Mexico-2007/lang.es-es/?Itemid=

Asociación de Internet. "Estudio sobre los hábitos de los usuarios de internet en México 20012" (2013). Consultado el 04 de junio 2019. https://www.asociaciondeinternet.mx/es/component/remository/Habitos-de-Internet/Estud io-sobre-los-habitos-de-los-usuarios-de-internet-en-Mexico-2012/lang.es-es/?Itemid= 
Asociación de Internet. "Estudio sobre los hábitos de los usuarios de internet en México 2018" (2018). Consultado el 04 de junio 2019 https://www.asociaciondeinternet.mx/es/component/remository/Habitos-de-Internet/14-Est udio-sobre-los-Habitos-de-los-usuarios-de-Internet-en-Mexico-2018/lang.es-es/?Itemid=

Ávila Yuriria, (2018). "Así asigna el INE el número de spots a los que cada partido y candidato tienen derecho". https://www.animalpolitico.com/2018/04/asi-asigna-el-ine-el-numero-de-spots-a-los-que -cada-partido-y-candidato-tienen-derecho/

Beatriz Fernández, Carmen. (2018). “Ciberpolítica 2018: tendencias en latinoamérica”. En: “Las campañas políticas electorales en América Latina”. Konrad-adenauer-stiftung e. V. https://dialogopolitico.org/w p-content/uploads/2018/11/Campa\%C3\%B1as-electorales-en-Am\%C3\%A9rica-Latina_WEB.pdf

Chávez Gabriela, (2017). “Las mentiras políticas son un negocio”. Expansión. https://expansion.mx/tecnolo gia/2017/11/03/las-mentiraspoliticas-son-un-negocio.

De Mauleón, Héctor, (2012). "YoSoy132 y el uso político de las redes sociales” en: Revista Nexos, núm. 417. México, Ed, Kathya Millares.

Deibert Ronald J, (2013). "Black Code, Inside the Battle for Cyberspace". McClelland \& Stewart. Toronto.

Esteinou, Javier, (2019). "Las “benditas” redes sociales y la victoria de AMLO/Morena”. Siempre. http://ww w.siempre.mx/2019/04/las-benditas-redes-sociales-y-la-victoria-de-amlo-morena/

Islas, Octavio, y Pimienta, Xóchitl, (2016). El empleo de redes sociales en las campañas presidenciales en México, 2012. España: Cuadernos artesanos de comunicación/100. ISBN -13: 978-84-16458-43-1. htt ps://dialnet.unirioja.es/servlet/libro?codigo $=581633$.

Islas, Octavio, (2018).“De bots, trolls y elecciones”. El Universal. https://www.eluniversal.com.mx/columna /octavio-islas/techbit/de-bots-trolls-y-elecciones

Lawrenece Lessig, ( 2006). “Code”. Ed. Basic Books. New York.

María Elena Meneses Rocha y Jacob Bañuelos Capistrán, (2009).“Internet y campañas electorales en México. La oportunidad postergada”. México: Breviarios de Cultura Política Instituto Electoral del Estado de México. IEEM. .

Martínez, Pario, (2012). “No se aceptará la imposición de Peña Nieto: \#YoSoy132”. Animal Político. https:// www.animalpolitico.com/2012/07/no-se-aceptara-la-imposicion-de-pena-nieto-yosoy132/

Morozov, Evgeny, (2011).“The Net Delusion: The Dark Side of Internet Freedom”. Public Affairs, New York. ISBN 978-1-58648-874-1

Navarro, María Fernanda, (2019) “Ellos son los creativos que dieron un vuelco a la campaña de AMLO"..http s://www.forbes.com.mx/ellos-son-los-creativos-que-dieron-un-vuelco-a-la-campana-de-amlo/

Nicolai Aldo, et.al. (2018). "Verificado.mx: Candidatos gastan más de 106 mdp en propaganda en internet, pero no declaran todos los anuncios". Animal Político. https://www.animalpolitico.com/2018/06/ver ificado-candidatos-anuncios-internet/

Norris, Pippa, (2002). "La participación ciudadana: México desde una perspectiva comparativa”. Harvard University.

Peinado, Fernando, (2018). "Las redes rotas de la campaña electoral en México". El País. https://elpais.com /internacional/2018/03/21/actualidad/1521628738_165398.html.

Recillas, Angélica, (2018). La acción y reacción de los bots de Morena" Etcétera. https://www.etcetera.com .mx/opinion/accion-reaccion-bots-trolls-morena/

Ricardo, (2018). “La publicidad digital wink ;) wink ;) wink ;)...”. Forbes. https://www.forbes.com.mx/pub licidad-digital-wink-wink/

Robertson, Jordan, Et. Al. (2016). “Cómo hackear una elección?”. Bloomberg Businessweek. https://www. bloomberg.com/features/2016-comomanipular-una-eleccion/. 
Rodríguez Adriana. (2018) "AMLO, el rey de las redes sociales durante el proceso electoral 2018".https://www.economiahoy.mx/elecciones-mexico-2018/noticias/9231206/06/18/ AMLO-el-rey-de-las-redes-sociales-durante-el-proceso-electoral-2018.html

Ronfeldt David, (1991). "Cyberocracy, Cyberspace and Cyberology: Political effects of the Information Revolution". RAND. California.

SonTusDatos, Artículo 12, A.C. (2018).“Datos personales e influencia política: Investigación sobre las estrategias digitales del Partido Revolucionario Institucional en 4 de sus campañas electorales recientes en México “.

Torres Soriano, Manuel, (2013). "Internet as a Driver of Political Change: Cyber- Pessimist and CyberOptimists”. Revista del Instituto Español de Estudios Estratégicos. Núm. 1.

Verificado MX https://verificado.mx/que-es-verificado-2018/

NOTAS

$1 \quad$ El programa se instala en los dispositivos de la persona vigilada (teléfono celular, ordenador) haciéndose pasar por un programa legítimo; una vez que FinFisher se aloja en el dispositivo, le da control remoto al atacante, permitiéndole grabar conversaciones; tener acceso a los archivos guardados; descargar listas de contactos, correos electrónicos, mensajes de texto, entre otros contenidos. FinFisher también puede intervenir la cámara web y el micrófono del aparato infectado.

2 Google no puede establecer relaciones contractuales con partidos políticos ni entidades que tengan alguna obligación legal según lo estipulado en la normativa de auditorías del Instituto Nacional Electoral de México. Tampoco puede aceptar pagos de dichos partidos ni entidades. Si tu entidad debe cumplir con alguna obligación conforme a dicha normativa, debes usar un intermediario para realizar las compras (por ejemplo, una agencia publicitaria). En Blanco, Ricardo (2018, marzo 22).

3 Estas son: la Ley General de Protección de Datos Personales en Posesión de Sujetos Obligados (LGPDPPSO) y la Ley Federal de Protección de Datos Personales en Posesión de Particulares (LFPDPPP) 